\title{
APPRENDRE UNE LANGUE ET DEVENIR BILINGUE : UN ECLAIRAGE ACQUISITIONNISTE SUR LES CONTACTS DE LANGUES
}

Bernard Py

Université de Neuchâtel

Apprendre et/ou faire usage d'une nouvelle langue, c'est créer ou gérer des contacts, et ceci dans plusieurs sens à la fois : contacts entre L1 et L2, contacts entre des apprenants et des locuteurs natifs monolingues de L2, contacts entre des cultures différentes... Il y a là incontestablement un terrain intéressant pour l'étude des contacts de langues en général. L'objectif de cet article est de dresser un inventaire plutôt personnel et partiel de travaux et de propositions qui nous paraissent pertinents du point de vue du thème de ce numéro, afin d'enrichir ainsi, tant soit peu, les références ou la boîte à outils de l'étude des contacts. Nous allons essayer de parcourir à grandes enjambées le chemin qui part du degré zéro du contact, c'est-à-dire de la notion abstraite d'exposition d'un apprenant à une langue, chemin qui mène à une vision tenant compte de manière plus concrète de la complexité des contextes sociaux et des opérations cognitives et pragmatiques qui interviennent dans le devenir bilingue (ou plurilingue). Notre contribution aura un caractère très synthétique, mais elle ne sera en aucun cas ni exhaustive ni encyclopédique.

Les recherches sur l'appropriation d'une nouvelle langue, le bilinguisme et les contacts de langues au sein d'une communauté politique, géographique ou culturelle sont longtemps restées confinées dans leurs territoires traditionnels respectifs. Nos ${ }^{1}$ intérêts et nos problèmes méthodologiques nous ont pourtant conduits à travailler simultanément et de manière complémentaire sur ces trois domaines. Nous avons été séduits par leur fécondation mutuelle: comme souvent, le passage d'une frontière ouvre l'esprit. Aux questions habituelles «comment apprend-on une nouvelle langue?», "comment s'articulent deux langues intégrées dans les ressources verbales d'une même personne? », ou encore «que se passe-t-il lorsque deux langues circulent au sein d'une même communauté sociale? » succède une question plus globale, à savoir «comment devient-on bilingue lorsque l'on est immergé dans une communauté alloglotte ou diglossique particulière, que l'on y pratique la communication bilingue et / ou exolingue ${ }^{2}$ et enfin

\footnotetext{
${ }^{1}$ Nous désignera ici l'équipe à géométrie variable qui, entre les Universités de Bâle, Lausanne et Neuchâtel, a développé entre 1984 et 2000 une approche collective et globale de ces trois domaines dans des recherches sur les migrations et les contacts de langues. Voir Lüdi, Py \& al. (1995), Lüdi \& Py (2003), ainsi que Py \& al. (2000).

${ }^{2}$ Porquier (1984), De Pietro (1988), Ishikawa (2002), Vasseur (2005), etc.
} 
que l'on y occupe une position socioculturelle déterminée ?» En d'autres termes, nous sommes d'avis que l'appropriation d'une nouvelle langue s'effectue selon un ensemble complexe de modalités particulières du processus général de contact, et que l'étude de cet ensemble permet d'en mieux observer et comprendre le processus général ${ }^{3}$.

Les études sur l'appropriation d'une nouvelle langue conduisent le chercheur à dégager ou à mettre en lumière des pertinences qui, sans être nécessairement propriété exclusive de cet objet particulier d'étude, paraissent néanmoins y jouer un rôle essentiel (il s'agit d'un cas parmi d'autres d'effet de loupe), et devraient par conséquent être prises en compte par une théorie générale du langage. Pour prendre un exemple l'asymétrie des ressources verbales et gestuelles de deux interlocuteurs (à savoir la communication dite exolingue) est un aspect tout à fait central lorsque l'on étudie des processus d'appropriation ou des situations de contact. Or cet aspect est le plus souvent ignoré dans les modèles les plus connus de la conversation. Nous admettons ainsi que ces situations constituent des modalités de contact et des lieux d'observation intéressants du point de vue méthodologique et théorique. Enfin, nous considérons que l'acquisition d'une L2 est déjà en soi une pratique bilingue (devenir bilingue, c'est déjà d'une certaine manière être bilingue) et que ce qu'on appelle bilinguisme est une position intermédiaire, plus ou moins stabilisée, située quelque part sur une trajectoire biographique ${ }^{4}$.

Cette notion de stabilisation suppose une dialectique entre deux représentations de la langue : d'un côté la langue comme ensemble de connaissances stabilisées, immobiles; de l'autre la langue comme pratique, ou ensemble dynamique de connaissances en mouvement, toujours ouvertes au changement. Les arguments en faveur du mouvement proviennent surtout de l'analyse du discours, alors que les arguments en faveur de la stabilisation proviennent plutôt de la recherche sur l'appropriation des langues étrangères (RALE). L'argument de l'analyse du discours s'appuie sur l'idée que le sens se construit de manière dialogique dans des interactions verbales toutes différentes les unes des autres ${ }^{5}$. Les arguments en faveur de l'immobilité viennent notamment de la didactique et de la recherche sur l'acquisition. En substance, ils partent du constat que la mémorisation (en tant que constitution de ressources verbales et gestuelles dans une nouvelle langue) suppose une certaine forme de stabilité. Un des problèmes de l'apprenant consiste justement en effet à décontextualiser des segments discursifs saillants, c'est-à-dire à prélever du matériel verbal ou gestuel vivant dans un discours en L2, de l'en extraire, de le traiter puis d'en faire un item stable au sein de ses propres ressources, prêt à un éventuel emploi dans un nouveau contexte ${ }^{6}$. En fait, ce processus comporte à la fois un effort de stabilisation (décontextualisation) et un effort de déstabilisation (au moment de la recontextualisation). Nous avons consacré beaucoup de temps à l'étude de ce processus, à travers une approche conversationniste de la SPA (séquence potentiellement acquisitionnelle). Cette expression désigne «une séquence conversationnelle exolingue qui rassemble, sur un nombre restreint de tours de parole, des formes discursives interprétables comme traces d'opérations cognitives constitutives d'un apprentissage de la langue dans laquelle se déroule l'échange $»^{7}$. Les SPA sont des événements langagiers observables, situés dans leur contexte d'occurrence. Elles connectent autostructuration et hétérostructuration. L'autostructuration est un travail de construction d'un énoncé avec les seuls moyens déjà disponibles, alors que dans l'hétérostructuration le partenaire le plus fort intervient dans la construction de l'énoncé du partenaire plus faible. L'observation porte par exemple sur la

\footnotetext{
${ }^{3}$ Cette démarche est fort bien illustrée dans Véronique (1994).

${ }^{4}$ Nous avons présenté et discuté ces idées à de nombreuses occasions. Voir par exemple Lüdi \& Py (1986) (nouvelle version en 2002), Lüdi \& Py (1995), Matthey (2003), Porquier \& Py (2004), ou Gajo \& al. 2004. Voir aussi Bange \& al. (2005) ainsi que Vasseur (2005).

${ }^{5}$ Voir notamment les travaux de Uli Dausendschön-Gay, Elisabeth Gülich ou Lorenza Mondada.

${ }^{6}$ On trouvera d'excellents exemples dans Montredon (2005).

${ }^{7}$ Porquier \& Py (2004 : 34). Voir aussi une partie des textes réunis par Gajo \& al. (2004).
} 
sollicitation de matériel lexical et son traitement interactif, puis sur les traces éventuelles du traitement qui aboutit à une saisie d'une nouvelle ressource ${ }^{8}$.

Le plus souvent l'étude des contacts de langues s'était cependant limitée à inventorier des variantes de contact et de comparer des grammaires descriptives des langues concernées. Pour l'analyse contrastive des années soixante et soixante-dix (motivée surtout par des objectifs pédagogiques), le contact entre L1 et L2 se réduit alors à une confrontation des systèmes respectifs envisagés dans une globalité abstraite et figée. Le résultat dépend évidemment beaucoup du modèle linguistique utiliséé. Les modalités de cette mise en contraste des systèmes sont définies par le linguiste lui-même, dans le meilleur des cas sur la base d'une analyse des erreurs ou d'une expérience pédagogique ou personnelle, ou encore sur de simples croyances. Ces modalités sont en principe fondées sur une rationalité linguistique dictée par la théorie de référence, mais elles ne coïncident pas nécessairement avec l'organisation psycholinguistique effective des connaissances des apprenants ni sur leur conduite discursive.

Par exemple on expliquera l'accent allemand d'un germanophone parlant français par le poids de la différence entre les règles qui définissent la place de l'accent tonique en allemand et en français. Cette interprétation comporte de toute évidence une part de vérité, mais on peut aussi recourir à des données relatives aux attitudes ou aux représentations des langues prises en considération, ou encore aux circonstances particulières de la communication ou au genre de discours. Pour certains apprenants, l'accent allemand aura avant tout une valeur identitaire. Adopter l'accent d'autrui, c'est dans une certaine mesure endosser une identité et occuper une place perçues par l'apprenant lui-même et/ou ses partenaires natifs, comme une sorte d'usurpation ou de tromperie socioculturelles.

$\mathrm{Ou}$, pour prendre un autre exemple, emprunté à une personne bilingue français / espagnol, un énoncé comme elle a appelé ta sour (pour ta sœur a appelé) peut certes être interprété comme un calque syntaxique opéré sur le modèle de ha llamado tu hermana, mais aussi certainement comme surgénéralisation de la tendance du français oral à utiliser systématiquement le pronom sujet devant la forme verbale (ta sœur elle a appelé).

S'ils ne sont pas «vaccinés» par leurs interlocuteurs monolingues ou par des procédés didactiques $a d$ hoc, les bilingues (c'est-à-dire les personnes pratiquant de manière régulière L1 et L2) seraient en quelque sorte manipulés à leur insu par les systèmes eux-mêmes. Ils ne seraient que les jouets inconscients et involontaires de systèmes que les circonstances obligent à cohabiter. On remarquera au passage la défiance implicite de cette théorie envers le bilinguisme en général, défiance reposant essentiellement sur deux préjugés d'un autre âge ${ }^{10}$ :

1. L'être humain serait constitué de manière à rendre possible un connaissance approfondie d'une seule langue, de telle sorte que toute implantation d'une deuxième ou nième langue ne pourrait qu'empiéter sur l'espace mental réservé à la première et ainsi la mettre en danger.

2. Toute langue serait liée étroitement à une même culture. Or l'être humain ne saurait vivre que dans une seule et même culture. L'implantation d'une nouvelle langue (et donc d'une autre culture) ne pourrait aboutir qu'à une catastrophe identitaire.

\footnotetext{
${ }^{8}$ Pour des exemples, consulter les publications citées en bibliographie.

${ }^{9}$ Le modèle structuraliste classique rend d'ailleurs la comparaison impossible, dans la mesure où les unités de la langue sont définies par l'ensemble du système, ce qui empêche de poser l'identité de deux formes appartenant à deux systèmes différents : il ne peut y avoir que des différences.

${ }^{10}$ Pour une discussion approfondie, voir Grosjean (1982), ou les travaux de Claire Blanche Benveniste et de Françoise Gadet sur le français oral.
} 
Cette mise en regard de langues en contact suppose quelques conditions théoriques relatives à la notion de système. Si l'on donne à cette notion son sens classique, on se met dans une situation difficile. Pour le structuralisme pur et dur en effet, aucun des éléments d'un système ne saurait coïncider avec un élément apparemment identique de l'autre système. Chaque élément se définit en effet comme expression linguistique par son environnement dans un système particulier. C'est la raison pour laquelle une voyelle nasale du français par exemple n'a en principe rien en commun avec une voyelle nasale du portugais, même si elle possède les mêmes traits acoustiques. C'est d'ailleurs pourquoi la traduction littérale mot à mot conduit le plus souvent à l'échec.

Depuis lors plusieurs changements épistémologiques se sont produits, en ce sens d'abord que le sujet épistémique est devenu énonciateur, que la place de cet 'énonciateur' a été reconnue, étendue et étoffée, reprise dans une perspective dialogique. C'est ainsi que Weinreich a, dès 1953, attiré 1'attention des chercheurs sur le fait que les langues entrent en contact non pas en un lieu abstrait, mais chez chaque individu concerné. En d'autres termes, c'est une personne ou un groupe bilingue ${ }^{11}$ qui est responsable de chaque événement de contact, ainsi que de ses incidences grammaticales. Ou encore : la personne bilingue n'est pas un simple réceptacle cognitif : elle est actrice en ce sens que c'est elle qui médiatise le contact entre les deux langues, et ceci toujours dans un certain contexte socioculturel. La pragmatique et la sociolinguistique, couplées à la cognition, jouent maintenant un rôle crucial dans cet enrichissement de la représentation que nous nous faisons du sujet acteur et énonciateur (Bange \& al. 2005).

Par ailleurs, nous avons montré ci-dessus que la recherche sur l'appropriation des langues étrangères (RALE) ne se contente plus des notions abstraites d'exposition à la langue cible, notion inspirée des travaux de Chomsky, ni de la notion de système ${ }^{12}$. Cette exposition a été traitée longtemps comme source d'alimentation (input) d'une boîte noire dont la fonction serait de «produire » une nouvelle compétence en L2. Pour de nombreux chercheurs des années quatrevingt, l'acquisition était le résultat en quelque sorte magique de l'exposition d'une personne à une langue, sous deux conditions certes : attitude positive face à L2 et accès à un sens minimal ${ }^{13}$. Cette position est insuffisante dans la mesure où ni la nature ni les circonstances de l'exposition ne sont prises en compte ${ }^{14}$. Or il paraît évident que ce n'est pas la même chose d'écouter un chanteur en L2 tout en conduisant, ou d'engager une relation affective avec une inconnue parlant L2.

Le contact est donc avant tout une activité sociale engagée dans un certain contexte autant ou plus qu'un état stabilisé. Or toute activité poursuit un objectif et se déroule dans un contexte dont elle est indissociable. Elle est aussi changement. Dans le cas du bilinguisme, ce changement peut être interprété comme acquisition, c'est-à-dire comme appropriation de nouvelles formes et de nouveaux schèmes syntaxiques, gestuels et discursifs. Il y a chez l'apprenant et/ou son partenaire une tension didactique qui donne aux échantillons de L2 (données) un statut de formes à s'approprier. En l'absence de tension, ces échantillons constituent un simple répertoire de ressources au service de diverses tâches de communication. On n'oubliera pas non plus que le changement ne se fait pas toujours dans la direction de L2 : il aboutit parfois à la création de variantes de contact, qui se situent à la périphérie, voire à 1'extérieur des systèmes L1 ou L2. Et comme tout changement, l'appropriation n'entraîne pas nécessairement la suppression des formes précédentes, mais souvent l'émergence de simples variantes. Elle entraîne avec elle également des persistances structurelles comme dit Nicolaï (2006).

11 Conformément à la tendance dominante aujourd'hui chez les chercheurs, nous appelons bilingue (ou plurilingue) toute personne qui est amenée à utiliser deux (ou plusieurs) langues de manière régulière.

12 Pour certains chercheurs, l'appropriation se contente de la simple immersion du sujet dans un bain de langue.

${ }^{13}$ Voir p. ex. Krashen (1985).

${ }^{14}$ Pour une prise en compte de ces circonstances, voir en particulier les travaux conduits autour du Groupe de Recherche sur l'Amérique Latine (GRAL). Par exemple Porquier (1994), 1995, Arditty \& Vasseur (2002), Perdue (1993), ou dans un cadre un peu différent Nicolaï (2006). 
Ce genre de raisonnement a conduit certains chercheurs (dont nous sommes) à contester les notions de compétence ou de système. La capacité d'un apprenant à intervenir dans une conversation exolingue tient dans une large mesure à des inférences fondées sur sa représentation de l'événement en cours, de son interlocuteur, du contexte, combinée avec l'ensemble de ses ressources. La notion de ressource nous paraît plus appropriée que celle de compétence, déjà surchargée de définitions hétéroclites provenant aussi bien de la grammaire générative que de la psycholinguistique ou de la didactique. Le terme de ressource ne contient aucun préjugé sur la nature exacte, le mode d'organisation et le degré d'homogénéité de son contenu. Ces inférences exploitent la marge d'approximation qui est si essentielle au langage. Essentielle parce qu'elle permet aussi la créativité, l'interprétation, l'adaptation au contexte, l'usage de ressources en phase de construction (qui est donc bien une condition sine qua non de l'interaction exolingue).

Dans cette perspective le bilinguisme se définit par un accès à des ressources issues de deux langues. Ni la quantité ni la qualité de celles-ci n'interviennent alors dans la reconnaissance du bilinguisme de quelqu'un. L'important est l'accessibilité à des ressources appropriées. Elles se caractérisent par leur disponibilité, leur hétérogénéité, leur légèreté leur utilité du point de vue de la construction de discours. Les notions de microsystème et de clause ${ }^{15}$ paraissent répondre à ces conditions.

L'expérience montre que la diversité des voies d'appropriation ne peut pas se ramener à un type unique. On observe au contraire une multitude hétérogène de types. D'où les notions de profil d'apprenant, de stratégies ou d'apprentissage situé, qui ont pour fonction d'expliquer une des origines possibles des différences observées dans le choix de telle ou telle voie d'appropriation, ou dans le rythme de progression. Le profil individuel est plus ou moins favorisé par le contexte, ou plus ou moins adapté. On observe par exemple dans l'enseignement et l'apprentissage des langues les phénomènes suivants :

1. L'école a tendance à accorder une priorité à la microsyntaxe au dépend de la macrosyntaxe. En d'autres termes, l'école valorise la connaissance des règles relatives à la rection des syntagmes au sein de la phrase, ainsi que la maîtrise de la morphologie associée à cette rection : il est capital pour l'évaluation d'un élève d'allemand, qu'il respecte par exemple la dislocation du syntagme verbal composé. L'organisation du discours est en revanche négligée lorsqu'il y a des problèmes par exemple de coréférence ou de gestion de l'énonciation (polyphonie par exemple). On favorise de la sorte les élèves «naturellement » attentifs à la morphologie ou à la rection, alors que l'immersion dans la vie sociale exige avant tout des ressources langagières et gestuelles suffisantes à l'accomplissement de la communication. On pense aux tables de conjugaison ou de déclinaison, à l'accord de l'adjectif, à la place du verbe, etc.

2. On accorde en général beaucoup d'importance à la mémorisation du lexique. Pour beaucoup d'élèves (ou d'anciens élèves), savoir une langue c'est connaître son vocabulaire. La notion de mot permet d'appréhender assez aisément le langage et d'en faire un thème de discussion. Les mots occupent une place de choix dans les représentations sociales courantes. Ils permettent souvent d'ébaucher un énoncé interprétable dans une langue mal connue. Dans ce cadre, l'apprentissage d'une nouvelle langue est perçu comme une opération d'étiquetage d'un monde préexistant au langage. On remarquera que l'entrée dans le lexique présente deux possibilités. D'abord la décontextualisation, qui consiste à extraire une expression de son contexte pour la stabiliser et en faire un objet de mémorisation.

\footnotetext{
${ }^{15}$ Nous avons empruntés ces notions respectivement à Gentilhomme (1980) et à Berrendonner \& ReichlerBéguelin (1989). Nous avons développé cette question dans divers articles, notamment dans Gajo \& al. (2004), ainsi que Py (2003).
} 
Ensuite la (re)contextualisation qui consiste à commencer par la mémorisation d'une expression déjà décontextualisée (par exemple dans une liste de mots), puis en essayant de l'utiliser à des fins de communication ${ }^{16}$.

3. Alors que les deux stratégies ci-dessus sont très répandues dans les écoles, l'apprentissage «naturel» favorise plutôt une organisation macrosyntaxique du discours. A partir d'énoncés constitués d'une expression simple déviantes d'un point de vue phonologique, morphologique, lexical, etc., enchaînées de manière parataxique, on observe une complexification progressive sur les plans macrosyntaxique puis microsyntaxique. L'organisation microsyntaxique paraît suivre plutôt que précéder ou accompagner l'organisation macrosyntaxique.

4. Le milieu social véhicule des représentations et des attitudes par rapport auxquelles les individus devront se situer, soit en y adhérent, soit en prenant plus ou moins de distance. L'objet de ces représentations ou attitudes concerne souvent la langue de manière indirecte, en ce sens qu'elles parlent plutôt des locuteurs d'une langue plutôt que de cette langue elle-même. Quoi qu'il en soit, elles jouent manifestement un rôle dans la construction ou le développement du bilinguisme. Par exemple de nombreux Suisses romands sont d'avis que «les dialectes alémaniques ne sont pas de vraies langues, car ils n'ont pas de grammaire ». Et quand un journaliste dit d'une politicienne «ce n'est pas une zurichoise arrogante, elle a de l'humour », il présuppose que, aux yeux de ses lecteurs sinon aux siens propres, la normalité des Zurichois est l'arrogance.

Comme tout ce qui a trait au langage, le contact d'apprentissage a donc bien des dimensions individuelles et sociales. Elles ne se manifestent pas tellement comme cadre extérieur ou comme scène, mais plutôt comme ingrédients des opérations cognitives et discursives. Elles interviennent souvent comme préférences plutôt que comme le résultat mécanique et incontournable de contraintes. L'activité de médiation entre les langues assurée par l'énonciateur prétend construire et transmettre du sens, c'est-à-dire pratiquement déclencher et orienter chez un interlocuteur une activité d'interprétation aussi proche que possible de l'intention de l'énonciateur. Cette dimension individuelle et sociale se matérialise par des interactions verbales de toutes sortes. En outre, une partie importante du travail d'appropriation est effectuée de manière collective. De ce point de vue, la relation verbale enseignant / enseigné est une forme simplifiée des interactions sociales plus complexes qui définissent le territoire de l'apprenant ${ }^{17}$.

\section{Références}

Arditty, Jo \& Mittner, Michèle (éds.), 1980. Acquisition d'une langue étrangère, Encrages, Paris : Université de Paris VIII.

Arditty, Jo \& Vasseur, Marie-Thérèse. 2002. Contextes, variabilité et activités d'appropriation des langues. Discours, action et appropriation des langues éd. par Francine Cicurel \& Daniel Véronique, 251-264. Paris : Presses de la Sorbonne-Nouvelle.

Bange, Pierre \& al. 2005. L'apprentissage d'une langue étrangère. Cognition et interaction. Paris : L'Harmattan.

\footnotetext{
${ }^{16}$ Voir Porquier \& Py (2004).

${ }^{17}$ Merci à Jacques Montredon, dont les remarques ont contribué à notre réflexion, notamment à propos de la notion de ressource.
} 
Berrendonner, Alain \& Reichler-Béguelin, Marie-José. 1989. Décalages : les niveaux de l'analyse linguistique. Langue française $81: 99-124$.

Cavalli, Marisa \& Coletta, Daniela \& al. 2002. Langues, bilinguisme et représentations sociales au Val d'Aoste. Aoste : Istituto Regionale di Ricerca Educativa della Valle d'Aoste.

Dausenschön-Gay, Ulrich, Gülich \& Elisabeth, Krafft, Ulrich. 1991. Linguistische Interaktionsanalysen. Beiträge zum 20. Romanistentag Freiburg (1987). Tübingen : Niemeyer.

De Pietro, Jean-François. 1988. Vers une typologie des situations de contact linguistique. Langage et société 43 : 65-89.

Gajo, Laurent \& al. 2004. Parcours au contact des langues. Textes commentés de Bernard Py. Paris : Didier.

Gentilhomme, Yves. 1980. Microsystèmes et acquisition des langues. Acquisition d'une langue étrangère, Encrages, éd. par Jo Arditty \& Michèle Mittner, 79-84. Paris : Université de Paris VIII

Grosjean, François. 1982. Life with two languages. Harvard: Cambridge University Press.

Ishikawa, Fumiya. 2002. L'interaction exolingue. Analyse de phénomènes métalinguistiques. Yokohama : Shumpushao.

Krashen, Stephen D. 1985. The input hypothesis: issues and implications. London : Longman.

Lüdi, Georges \& Py, Bernard. 2003 [1986]. Etre bilingue. Berne : Lang, $3^{\text {ème }}$ édition revue.

Lüdi, Georges \& Bernard Py \& al. 1995. Changement de langage et langage du changement. Aspects linguistiques de la migration interne en Suisse. Lausanne : L'Age d'homme.

Matthey, Marinette. 2003. Apprentissage d'une langue et interaction verbale. Berne : Peter Lang, $2^{\text {ème }}$ édition.

Matthey, Marinette (éd.). 1997a. Les langues et leurs images. Neuchâtel : Institut de recherche et de documentation pédagogique (IRDP).

Mondada, Lorenza. 1995. La construction discursive des objets de savoir dans l'écriture de la science, Réseaux 71 : 55-77.

Montredon, Jacques. 2005. De bouche à oreille. Dictionnaire des étudiants étrangers de Besançon. Besançon : Cêtre.

Moore, Danièle (éd.). 2001. Les représentations des langues et de leur apprentissage. Références, modèles, données et méthodes. Paris : Didier.

Nicolaï, Robert. Sous presse. La vision des faits. Paris : L’Harmattan.

Perdue, Clive (éd.). 1993. Adult second language acquisition: cross-linguistic perspectives. Vol. I: Field methods. Vol. II: The results. Cambridge: Cambridge University Press.

Porquier, Rémy. 1984. Communication exolingue et apprentissage des langues. Acquisition d'une langue étrangère III. Actes du colloque organisé les 16-18 septembre 1982 à l'Université de Neuchâtel éd. par Bernard Py, 17-47. Paris : Encrages. 
Porquier, Rémy. 1994. Communication exolingue et contextes d'appropriation : le continuum acquisition/apprentissage. Bulletin suisse de linguistique appliquée 59 : 159-169.

Porquier, Rémy. 1995. Trajectoires d'apprentissage(s) de langues: diversité et multiplicité des parcours. Etudes de linguistique appliquée 98 : 92-102.

Porquier. Rémy \& Py, Bernard. 2004. Apprentissage d'une langue étrangère : contextes et discours. Paris : Didier.

Py, Bernard. 1980. Quelques réflexions sur la notion d'interlangue. Travaux neuchâtelois de linguistique (TRANEL) $1:$ 31-54. Repris dans Laurent Gajo \& al. (éds.). 2004. 25-39.

Py, Bernard \& al. 2000. Analyse conversationnelle et représentations sociales. Unité et diversité de l'image du bilinguisme. Travaux neuchâtelois de linguistique (TRANEL) 32 : 39-53.

Py, Bernard. 2003. Acquisition d'une langue seconde, organisation macrosyntaxique et émergence d'une microsyntaxe. Marges linguistiques 4. www.marges-linguistiques.com.

Vasseur, Marie-Thérèse. 2005. Rencontres de langues. Question(s) d'interaction. Paris : Didier.

Véronique, Daniel (éd.). 1994. Créolisation et acquisition des langues. Aix-en-Provence : Presses Universitaires de Provence.

Weinreich, Uriel. 1953. Languages in Contact, The Hague: Mouton. 